\title{
Pratiques exemplaires du Réseau des laboratoires de santé publique du Canada relativement à la COVID-19
}

\section{Groupe de travail sur les infections virales respiratoires ${ }^{1}$}

\section{Résumé}

La capacité de détecter le coronavirus du syndrome respiratoire aigu sévère 2 (SRAS-CoV-2), l'agent étiologique de la COVID-19, est un élément fondamental des stratégies de contrôle et d'atténuation du Canada. La confirmation en laboratoire des cas de COVID-19 permet de prendre les mesures d'intervention appropriées en termes de traitement clinique et de santé publique. Le fait que l'objectif local est le contrôle ou l'atténuation dépend de l'épidémiologie locale de la pandémie. Le groupe de travail sur les infections virales respiratoires du Réseau des laboratoires de santé publique du Canada a mis au point des lignes directrices détaillées sur les pratiques exemplaires visant la détection du SRAS-CoV-2. Les pratiques exemplaires pour les prélèvements, le transport, le dépistage et la biosécurité sont abordées du point de vue des laboratoires de santé publique canadiens afin d'assurer une approche uniforme partout au pays :

1. Des tests de dépistage de COVID-19 basés sur une population devraient être effectués au départ à des fins de surveillance

2. Le prélèvement d'un écouvillon rhinopharyngé est l'échantillon de choix pour les tests de routine

3. Les tests d'amplification des acides nucléiques (comme la réaction en chaîne de la polymérase après transcription inverse en temps réel) sont la méthode privilégiée pour les tests de routine du SRAS-CoV-2

4. Il faut encourager la décentralisation des tests d'amplification des acides nucléiques pour la COVID-19 vers les hôpitaux ou d'autres laboratoires médicaux de complexité élevée pour accroitre la capacité d'effectuer les tests et répondre aux demandes croissantes

5. Dans les stades précoces de la pandémie, les tests positifs (environ 10 à 20) et négatifs (environ 50) par un laboratoire provincial doivent être confirmés au Laboratoire national de microbiologie

6. Il faut surveiller la co-circulation d'autres agents viraux associée aux maladies de type grippal (p. ex. le virus de la grippe $A$ et $B$ et le virus respiratoire syncytial) comme la capacité le permet, dans le cadre de la surveillance continue

7. Après avoir obtenu la validation, les tests sérologiques peuvent servir à évaluer la présence ou I'absence de réaction immunitaire au SRAS-CoV-2 soit au niveau de la population, soit au niveau individuel pour certaines indications, mais ceux-ci seront probablement peu utiles pour établir un diagnostic de la forme grave du COVID-19

Ces recommandations seront mises à jour à mesure que de nouveaux renseignements sont disponibles.
Cette oeuvre est mise à la disposition selon les termes de la licence internationale Creative Commons Attribution 4.0

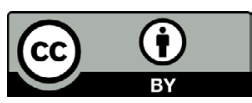

Affiliation

${ }^{1}$ Réseau des laboratoires de santé publique du Canada, Winnipeg, MB

\section{${ }^{\star}$ Correspondance :}

dionne.marcino@canada.ca

Citation proposée : Groupe de travail sur les infections virales respiratoires. Pratiques exemplaires du Réseau des laboratoires de santé publique du Canada relativement à la COVID-19. Relevé des maladies transmissibles au Canada 2020;46(5):127-33. https://doi.org/10.14745/ccdr.v46i05a02f

Mots-clés : COVID-19, pratiques exemplaires, Canada, tests, surveillance

\section{Introduction}

Depuis la déclaration de la maladie à coronavirus 2019 (COVID-19) causée par le SRAS-CoV-2 à la fin du mois de décembre 2019 à Wuhan, dans la province du Hubei en Chine, la vaste majorité des pays ont maintenant déclaré des cas de COVID-19 confirmés en laboratoire. Étant donné la propagation soutenue de la COVID-19, le 11 mars 2020, I'Organisation mondiale de la Santé a déclaré que la situation représentait une pandémie (1).

La présentation clinique de la COVID-19, qui est causée par le SRAS-CoV-2, est non spécifique et chevauche d'autres virus respiratoires saisonniers, y compris la grippe. La capacité à dépister le SRAS-CoV-2 chez les patients est cruciale pour la 
surveillance, le diagnostic et le traitement clinique des personnes qui présentent une maladie respiratoire aiguë, un syndrome grippal (SG) et une maladie respiratoire sévère afin d'appuyer les stratégies de contrôle et d'atténuation du Canada.

Le but des tests de dépistage du SRAS-CoV-2 peut entrer dans deux catégories générales, et dépendra de l'épidémiologie locale et des objectifs des stratégies de santé publique (contrôle ou atténuation) :

1) La conduite de tests dans le but de détecter les cas de forte probabilité chez les personnes présentant une maladie respiratoire aiguë et un SG et les critères d'exposition appropriés est essentielle pour veiller à ce que les cas de COVID-19 soient dépistés en temps utile afin de s'assurer que la prise en charge clinique et de santé publique puisse avoir lieu au cours de la phase de contrôle de la pandémie. De plus, lorsque les nombres locaux sont faibles, la conduite de tests appuiera probablement les stratégies agressives de dépistage des cas pour la recherche anticipée des contacts et la mise en œuvre de l'auto-isolement. Lorsque le virus devient généralisé, la conduite de tests sur des échantillons communautaires devrait être réservée aux programmes de surveillance communautaires, les autres tests mettant l'accent sur les patients hospitalisés avec une maladie respiratoire aiguë et ceux qui ont des facteurs de risque de maladie sévère où les résultats des tests peuvent influencer les décisions concernant les soins et le traitement, le contrôle de l'infection (y compris les éclosions), la prise en charge des contacts étroits, et le soutien aux collectivités éloignées. Il est important de ne pas retarder la prise des décisions susmentionnées sur la prise en charge et la prévention en attendant les résultats. II arrive que des cas de COVID-19 soient atteints de co-infections à d'autres virus, notamment la grippe. Il faut poursuivre le dépistage de la grippe chez les patients hospitalisés afin d'aider à appuyer la prise en charge des patients au moyen d'antiviraux.

2) Il faut assurer la surveillance basée sur une population pour continuer d'identifier les cas de COVID-19 et faciliter le suivi d'autres agents viraux, comme la para-influenza du virus respiratoire syncytial, l'adénovirus et les rhinovirus, qui co-circulent durant la saison de la grippe et à d'autres périodes de l'année.

Les présentes lignes directrices sur les Pratiques exemplaires devraient être utilisées conjointement avec les lignes directrices provinciales et territoriales pertinentes. L'Agence de la santé publique du Canada (ASPC) affichera régulièrement des mises à jour et des documents connexes (2).

\section{Surveillance}

La surveillance basée sur une population est importante aux différents stades de la pandémie de COVID-19. Les secteurs d'intérêt de la surveillance changeront à mesure que les priorités de dépistage s'ajustent lorsque le système de santé passe d'une phase de contrôle à une phase d'atténuation. Il est important de noter que la performance des technologies existantes pour le dépistage de la COVID-19 ne suffit pas comme outil de dépistage général auprès de la population, et que l'utilisation ciblée des tests dans les populations où la probabilité avant le test est plus élevée, où chez qui les bienfaits éventuels sont les plus élevés, demeure un principe important de la sélection d'échantillons.

Durant le contrôle, la surveillance basée sur une population est très importante, car il est possible que l'infection légèrement symptomatique de SRAS-CoV-2 joue un rôle dans la transmission communautaire. À ce stade, la majorité des patients testés pour le SRAS-CoV-2 sont ambulatoires, et peu des patients hospitalisés répondent aux critères d'exposition pour en faire un cas suspect (3). Durant cette période, il est important de mener un dépistage de surveillance auprès d'un sous-ensemble de personnes hospitalisées et des personnes vues en milieu ambulatoire qui ont une maladie respiratoire aiguë ou un SG mais aucun facteur de risque particulier pour la COVID-19. Une surveillance communautaire supplémentaire devrait avoir lieu dans les foyers de soins de longue durée, où la population de patients âgés, souffrant souvent de maladies concomitantes, présente le plus grand risque de complications et d'infection fatale. Cette surveillance pourrait se faire en testant une sélection appropriée (conformément aux directives des autorités de contrôle des éclosions) ou tous les échantillons d'éclosion respiratoire pour le SRAS-CoV-2.

Durant l'atténuation, il est présumé que le virus circulera de façon généralisée dans différents secteurs de la collectivité. Au cours de cette période, les tests de dépistage de la COVID-19 serviront à identifier les cas chez les patients hospitalisés, qui représentent les personnes les plus gravement malades. Les tests communautaires de dépistage du SRAS-CoV-2 seront offerts moins régulièrement aux patients ambulatoires, quoiqu'ils devraient se poursuivre pour les travailleurs de la santé ambulatoires qui ont un SG (et peut-être une maladie respiratoire aiguë), les éclosions institutionnelles, les collectivités éloignées et les collectivités confinées ou d'hébergement en commun, et ils peuvent être fournis pour les populations qui ont des facteurs de risque de maladie sévère (p. ex. âgées de 60 ans ou plus, présence de maladies concomitantes). Des lignes directrices particulières pour le contrôle, l'échantillonnage, les prélèvements et les tests seront élaborées par le système de soins de santé provincial local. II faut maintenir les programmes de surveillance ambulatoires durant la phase d'atténuation afin de fournir des données sur la prévalence du SRAS-CoV-2 dans la collectivité, ce qui appuiera le suivi des progrès de la pandémie.

Pour aider à maximaliser l'utilisation de données de tests en laboratoires pour permettre la surveillance de la COVID-19, les hôpitaux ou d'autres laboratoires de grande complexité qui conduisent les tests devraient produire des données sommaires sur les tests afin de compléter les données sur les tests effectués 
dans leurs laboratoires provinciaux de santé publique. Ces données peuvent alors aider à éclairer les aperçus locaux, provinciaux et fédéraux de l'activité pandémique. Les provinces devraient chercher à atteindre des volumes de surveillance et de tests de dépistage des cas adéquats, ce qui donnera un aperçu quotidien approximatif de la prévalence de la maladie dans leurs administrations. La détermination de ce volume minimum s'appuie sur plusieurs facteurs et devrait être effectuée en collaboration avec un soutien biostatistique ou épidémiologique.

Il faut aussi mettre en place une surveillance afin d'aider le contrôle global de l'épidémiologie moléculaire du SRAS-CoV-2. Elle permettra d'établir toute différence géographique des souches en circulation, et peut-être des variantes génomiques pertinentes sur le plan clinique. La surveillance moléculaire fournira aussi des données pour contribuer à la surveillance de toute incompatibilité d'amorce ou de sonde des méthodes diagnostiques avec le SRAS-CoV-2 qui pourrait nuire aux caractéristiques de performance des méthodes diagnostiques. Ces efforts doivent être coordonnés entre toutes les administrations et dirigés par des installations reliées à l'Organisation mondiale de la Santé comme le Laboratoire national de microbiologie (LNM) à Winnipeg. Même si une recherche plus poussée s'impose, il peut éclairer les questions sur l'immunité après infection et le potentiel de réinfection, en plus d'aider à la planification et à la conception de vaccins. Même s'il n'existe aucune thérapie antivirale particulière pour le SRAS-CoV-2, les données de séquençage génomique peuvent être utiles pour prédire les phénotypes résistants si des antiviraux efficaces sont développés.

Des études de séroprévalence peuvent aussi être effectuées pour aider à documenter les taux d'attaque de la population par la COVID-19 durant la pandémie. Ces études seraient effectuées au moyen d'une sérologie du SRAS-CoV-2 sur un ensemble représentatif de sérums résiduels de tous les groupes d'âge, et en répétant celle-ci à des intervalles établis au cours des prochains mois. La difficulté principale pour la tenue de cette activité réside dans le fait qu'aucune méthode d'analyse commerciale n'a encore été validée pour les tests cliniques, quoique des efforts de validation soient en cours au Canada, et I'utilité de telles analyses sur une échelle de population générale n'est pas encore confirmée.

\section{Tests de diagnostic}

Au cours de la période de contrôle, les efforts porteront sur le dépistage intense des cas afin d'assurer l'identification précoce, I'isolement précoce, le diagnostic précoce et le traitement précoce ainsi que la prise en charge appropriée des contacts et le suivi. Ces mesures viseront à la fois les milieux de patients externes (ambulatoires) et internes. Lorsque l'épidémiologie de l'éclosion laisse entendre que le contrôle n'est pas possible et que les ressources seront poussées à la limite, le laboratoire appuiera l'objectif d'atténuation et accordera la priorité des tests aux groupes de patients suivants : 1) les patients hospitalisés qui présentent tous les degrés de maladie respiratoire aiguë, y compris une maladie respiratoire sévère, le SG et une maladie respiratoire plus bénigne; 2 ) les patients pour qui les tests de dépistage aideront aux décisions concernant les soins, le contrôle de l'infection (y compris les éclosions) ou la prise en charge des contacts étroits; 3 ) les personnes décédées des suites d'une maladie aiguë soupçonnée d'avoir été causée par le virus de la grippe ou autre virus respiratoire comme le SRAS-CoV-2; 4) les travailleurs de la santé atteints d'une maladie respiratoire aiguë ou d'un SG; et 5) les personnes vivant dans des collectivités éloignées et isolées.

Dans la phase d'atténuation, lorsque la circulation virale dans la communauté est établie, les patients externes peuvent, occasionnellement seulement, faire l'objet de tests. Les algorithmes de dépistage spécifiques seront décidés par le système de santé de chaque province, probablement avec une orientation semblable à celle qui est décrite plus haut. Les tests de dépistage ne sont pas indiqués pour le traitement clinique des personnes atteintes d'une infection respiratoire non complexe résidant dans des collectivités où le SRAS-CoV-2 circule.

\section{Type de prélèvements}

Récemment, l'Organisation mondiale de la Santé a signalé que le SRAS-CoV-2 avait été détecté dans les prélèvements respiratoires, fécaux et sanguins (4). Les données préliminaires signalent la détection du virus dans les échantillons des voies respiratoires supérieures 1 à 2 jours avant l'apparition des symptômes, lesquels persistent de 7 à 12 jours dans les cas modérés et jusqu'à deux semaines dans les cas sévères. Le virus a été cultivé à partir des échantillons des voies respiratoires jusqu'à huit jours après l'apparition des symptômes. Bien que le SRAS-CoV-2 ait aussi été détecté dans la salive, l'emploi de celle-ci pour les tests de dépistage doit faire l'objet d'une étude plus poussée.

L'acide ribonucléique (ARN) viral a été détecté dans les matières fécales chez jusqu'à $30 \%$ des patients à compter du cinquième jour suivant l'apparition des symptômes, et persiste pendant un maximum de cinq semaines dans les cas modérés. Cependant, il n'est pas clair si cette présence indique l'excrétion du virus infectieux. Même si un virus vivant a été cultivé à partir des selles dans certains cas, le rôle de la transmission oro-fécale n'est pas encore bien compris.

En ce moment, les tests de dépistage visent principalement les prélèvements respiratoires. Les premières données laissent entendre que des charges virales moins élevées peuvent être détectées dans des prélèvements d'écouvillons rhinopharyngés que ceux des écouvillons de la gorge (5), et à ce titre, ils représentent les prélèvements des voies respiratoires supérieures à privilégier. De plus, il s'agit des prélèvements à privilégier pour la détection de la grippe, qui peut avoir une présentation 
clinique semblable. Le crachat est un prélèvement utile des voies respiratoires inférieures, et il peut être prélevé chez les patients qui ont une toux productive. Cependant, il n'est pas recommandé d'induire le crachat en raison du risque de production d'aérosols. On recommande les écouvillons floqués pour les prélèvements rhinopharyngés ou du nez et de la gorge.

\section{Autres instruments de prélèvement}

En cas d'interruption de la chaîne d'approvisionnement et d'une incapacité à obtenir des écouvillons floqués ou un support de transport viral, on peut envisager d'utiliser d'autres options comme de la rayonne sur un plastique ou des fils. Des solutions de rechange envisagées pour les milieux de transport de virus peuvent inclure une solution saline dans un tampon phosphate ou l'alcool pour assurer la stabilisation. Les écouvillons en bois sont considérés comme inhibiteurs pour les tests fondés sur l'acide nucléique, et ils ne sont donc pas recommandés, à moins de validation contraire. Tout autre instrument de prélèvement ou ensemble d'expédition devra être validé pour son utilisation dans les tests cliniques. De plus amples renseignements sur les trousses de prélèvement de rechange sont disponibles auprès de la Food and Drug Administration des États-Unis (6).

\section{Regroupement des prélèvements}

Le regroupement de plusieurs prélèvements peut être envisagé comme façon d'accroître la production durant les périodes de soumissions élevées, et afin de préserver les réactifs durant les périodes de pénuries. Si le regroupement est positif, chacun des prélèvements du regroupement doit être testé de nouveau pour déterminer lequel d'entre eux est positif. Lorsque les prélèvements sont regroupés, le compromis est une baisse de la sensibilité. Un laboratoire qui envisage le regroupement doit mener sa propre évaluation des répercussions sur la sensibilité, car cela sera propre à sa méthode d'analyse et au laboratoire. II pourra s'en servir pour décider du nombre optimal à regrouper dans leur milieu. Les travaux menés dans le cadre des éclosions de la grippe ont indiqué que la sensibilité est considérablement réduite si l'on regroupe plus de quatre prélèvements. Les laboratoires peuvent choisir de n'analyser que les prélèvements non critiques à l'aide d'un protocole de mise en réserve et de réserver les tests de prélèvement unique aux patients ayant une maladie plus sévère (p. ex. les patients hospitalisés). À mesure que la positivité augmente, le nombre de prélèvements du regroupement devra être réduit pour en assurer l'efficacité. En règle générale, lorsque le taux de tests positifs atteint la gamme des $8 \%$ à $10 \%$, il n'y a pas d'avantages à regrouper un nombre donné de prélèvements (tableau 1).

\section{Transport des prélèvements}

Les prélèvements devraient être transportés au laboratoire dès que possible, préférablement dans les 72 heures, sur blocs réfrigérants. Si l'on prévoit un délai plus long, il faut congeler
Tableau 1 : Types de prélèvements à privilégier et autres

\begin{tabular}{|c|c|c|}
\hline $\begin{array}{l}\text { Nature de } \\
\text { la maladie }\end{array}$ & Prélèvement à privilégier & $\begin{array}{c}\text { Autres } \\
\text { prélèvements }\end{array}$ \\
\hline $\begin{array}{l}\text { Maladie légère } \\
\text { à modérée } \\
\text { semblable à la } \\
\text { grippe }\end{array}$ & $\begin{array}{l}\text { Prélèvement d'un écouvillon } \\
\text { rhinopharyngé } \\
\text { Vous pouvez visionner le } \\
\text { prélèvement d'un écouvillon } \\
\text { rhinopharyngé à http:// } \\
\text { www.youtube.com/ } \\
\text { watch?v=TFwSefezlHU }\end{array}$ & $\begin{array}{l}\text { Prélèvement d'un } \\
\text { écouvillon de la cavité } \\
\text { nasale, écouvillon } \\
\text { de la gorge ou les } \\
\text { deux https://vimeo. } \\
\text { com/397169241 }\end{array}$ \\
\hline $\begin{array}{l}\text { Maladie } \\
\text { respiratoire } \\
\text { sévère }\end{array}$ & $\begin{array}{l}\text { Prélèvement d'un écouvillon } \\
\text { rhinopharyngé ET lavage } \\
\text { endotrachéal ou bronchoalvéolaire. } \\
\text { Crachat (en cas de toux } \\
\text { productive) }\end{array}$ & $\begin{array}{l}\text { Crachat, prélèvement } \\
\text { d'un écouvillon de la } \\
\text { gorge }\end{array}$ \\
\hline Autopsie & $\begin{array}{l}\text { Prélèvement d'un écouvillon } \\
\text { rhinopharyngé ET d'un écouvillon } \\
\text { de la gorge } \\
\text { Tissu pulmonaire ou autres } \\
\text { tissus d'un organe participant } \\
\text { soupçonné. Les prelèvements } \\
\text { devraient être frais ou congelés à } \\
-70^{\circ} \mathrm{C} \text { ou moins. Ne pas les placer } \\
\text { dans une solution de fixation à la } \\
\text { formaline }\end{array}$ & Sans objet \\
\hline
\end{tabular}

les prélèvements à $-70^{\circ} \mathrm{C}$ ou plus froid, et les transporter sur de la glace sèche. Cependant, les prélèvements ne doivent pas être congelés à $-20^{\circ} \mathrm{C}$, car cela risque de nuire à la récupération du virus si une culture est nécessaire. S'il n'est pas possible de les congeler à $-70^{\circ} \mathrm{C}$ ou moins ou sur de la glace sèche, les prélèvements devraient demeurer à $4^{\circ} \mathrm{C}$ et être expédiés dès que possible. Les prélèvements doivent être transportés comme prélèvements diagnostics définis par le Transport des marchandises dangereuses conformément à la pratique habituelle pour les prélèvements de la grippe saisonnière, et aucune précaution accrue n'est nécessaire. Consultez l'Avis de biosécurité SRAS-CoV-2 de l'ASPC pour en savoir plus (7).

Les éprouvettes de prélèvements devraient être étiquetées convenablement et la réquisition remplie correctement et intégralement, avec les noms et identifiants uniques du patient correspondant, ainsi que les renseignements cliniques ou de santé publique nécessaires.

\section{Méthodes de détection}

Bien qu'il existe d'autres méthodes de détection du SRAS-CoV-2, les méthodes de détection en laboratoires cliniques se limitent à la détection moléculaire à l'aide de tests d'amplification des acides nucléiques (TAAN) et de culture virale.

\section{Tests d'amplification des acides nucléiques}

Au moment de la publication des présentes, il y a un nombre croissant d'analyses commerciales pour la détection du SRAS-CoV-2. Beaucoup de laboratoires mettent en œuvre des tests internes développés en laboratoire fondés sur la détection d'ARN polymérase ARN-dépendante, les gènes enveloppes 
et de capside nucléique, alors que d'autres mettent en œuvre des analyses commerciales qui détectent diverses cibles virales. Certains laboratoires ont un TAAN ARN polymérase pan béta coronavirus, qui est ensuite confirmé par séquençage d'acides nucléiques, quoique la plupart des laboratoires ont adopté des méthodes en temps réel qui identifient directement deux cibles génétiques différentes - le séquençage génétique est réservé aux cas où une cible unique est indéterminée dans l'analyse de la réaction en chaîne de la polymérase après transcription inverse (rRT-PCR) et qu'une clarification approfondie du résultat de laboratoire est jugée cliniquement nécessaire.

En raison de l'évolution de l'éclosion en pandémie, et du fait que le SRAS-CoV-2 n'est plus un résultat rare des tests en laboratoire, la détection d'une cible unique en conditions bien validées suffit pour la confirmation en laboratoire du SRAS-CoV-2.

Bien qu'il y ait peu de données sur la performance diagnostique des TAAN actuels, selon les données préliminaires des laboratoires canadiens, le niveau des tests de détection est d'une excellente sensibilité (limite de détection de $95 \%$ inférieure à 10 copies par réaction) et spécificité analytiques. Pendant la validation du niveau des tests de détection, les laboratoires devraient déterminer la valeur maximale du seuil du cycle pour la détection des cibles, guidés par la limite de détection de $95 \%$ générée dans leur laboratoire. Ils devraient aussi décider si un écart indéterminé du cycle seuil pour cette analyse particulière est nécessaire, ainsi que les valeurs du cycle seuil à inclure dans l'écart indéterminé. Les patients dont le test est initialement négatif devraient être testés de nouveau si la suspicion clinique de COVID-19 demeure élevée, en particulier chez les patients hospitalisés qui ne répondent pas au traitement. II faut obtenir des échantillons des voies respiratoires inférieures auprès des patients qui démontrent des signes de pneumonie afin d'accroître la sensibilité clinique. La performance des tests chez les patients atteints de différentes sévérités de maladie (p. ex. asymptomatiques, maladie bénigne, hospitalisés) est susceptible de varier, et ces différences n'ont pas bien été caractérisées. II n'est pas recommandé d'effectuer des tests réguliers auprès des patients asymptomatiques. II sera important de mener une évaluation continue des tests disponibles commercialement à mesure qu'ils sont développés afin de caractériser leur performance dans le milieu clinique et tout au long de la pandémie. Les laboratoires de santé publique devraient prendre des mesures appropriées et aider à établir des sites de test supplémentaires dans leurs administrations respectives.

\section{Tests moléculaires au point de service}

Les méthodes de détection moléculaire commerciales sont autorisées par Santé Canada, et d'autres le deviendront, pour utilisation aux points de service $(P d S)$ à l'extérieur du laboratoire. Avant que des établissements au Canada envisagent d'utiliser un PdS ou un instrument non de classe III « à une autre fin que celle déclarée sur son étiquette " pour les tests près des PdS, il faudrait établir un plan de mise en œuvre et de qualité avec un microbiologiste clinique ou médical et un directeur médical de laboratoire approprié. Si possible, il faut établir un système provincial pour saisir les données produites par les tests aux PdS afin d'aider à la surveillance en laboratoire. Comme pour toute activité des laboratoires médicaux, le respect de toute information personnelle sur la santé appropriée, de tous les règlements et normes d'accréditation de laboratoire et d'autorisation de laboratoire médical doit être pris en considération avant d'offrir ces tests.

\section{Isolement du virus}

L'isolement du virus est limité aux laboratoires qui ont des capacités de niveau de confinement (NC) 3 autorisées, et ne jouera pas un rôle important dans le diagnostic des patients atteints de la COVID-19. II servira principalement à propager le virus pour la génération de matières de contrôle des ARN positifs nécessaires aux TAAN. Il peut également être nécessaire pour appuyer les méthodes sérologiques fondées sur la croissance si elles sont développées (p. ex. microneutralisation), le développement de vaccins et d'autres domaines de recherche.

\section{Sérologie}

Des méthodes de diagnostic sérologique sont développées, mais elles n'ont pas encore été introduites dans I'utilisation clinique de routine au Canada ou ailleurs. Plusieurs plateformes qui ciblent diverses immunoglobulines (lgM, $\lg G, \lg A$ ) et les anticorps totaux par rapport à différents antigènes du SRAS-CoV-2, comme la protéine de spicule et la protéine capside nucléique, sont disponibles à des fins d'évaluation. Selon la documentation disponible, la détection d'une réaction sérologique semble moins fiable dans la première semaine après l'apparition des symptômes où la sensibilité est faible. La sensibilité de la détection augmente au $14^{\mathrm{e}}$ jour après l'apparition des symptômes. La durée de la séropositivité après l'infection et la question de savoir si la réaction immunitaire offre une protection contre la réinfection ou s'y rapporte doivent être déterminées avant de pouvoir formuler une interprétation relativement à l'immunité.

Le rôle de la sérologie dans le diagnostic d'une maladie grave et la prise en charge du patient sera probablement d'une utilité limitée. Lorsque nous comprendrons mieux la dynamique des réactions sérologiques, il se peut que la sérologie joue un rôle dans ce qui suit : utilisation dans les études séroépidémiologiques afin de mieux comprendre la proportion non diagnostiquée de la population au fil du temps et fournir une estimation plus précise du taux d'attaque; une méthode complémentaire à la méthode rRT-PCR pour les tests diagnostiques des patients qui sont négatifs à la méthode rRT-PCR, vers la fin de leur maladie, et qui ont de grandes difficultés à gérer les contacts qui bénéficieraient de l'information fournie par une sérologie complémentaire; la mise en œuvre de mesures de contrôle et la gestion efficace des populations considérablement à risque, y compris pour évaluer leur statut sérologique; et une fois qu'un vaccin est disponible, elle peut servir à déterminer, auprès des populations à risque élevé, à qui la priorité doit être accordée pour la vaccination anticipée. 
Il existe actuellement deux modalités de dépistage sur le marché : les essais d'immuno-absorption enzymatique (ELISA) et les essais au PdS. Les caractéristiques de performance des deux modalités doivent être établies en particulier, la sensibilité, la spécificité, la valeur prédictive positive et la valeur prédictive négative, en plus de l'interprétation des résultats positifs.

Les méthodes ELISA peuvent faire l'objet d'un traitement de haute capacité, d'un contrôle et d'une assurance de la qualité appropriés, sont moins susceptibles à la subjectivité de l'opérateur en matière d'interprétation, et il est facile d'intégrer les rapports sur leurs résultats dans les systèmes d'information existants des laboratoires. Les méthodes ELISA peuvent aussi fournir des estimations quantitatives sur la quantité d'anticorps présente. Cependant, elles sont plus exigeantes en main-d'œuvre, exigent de l'équipement spécialisé, des réactifs et une expertise en laboratoire, et elles ne donnent pas de résultats rapides. En tant qu'estimation de la protection de la réaction immunitaire, les résultats ELISA devraient être comparés à ceux des essais de neutralisation du virus. Cependant, à I'heure actuelle, les essais de neutralisation ne sont pas produits sur le plan commercial et ne peuvent être utilisés que dans des laboratoires de complexité élevée qui peuvent effectuer la culture des tissus et la culture virale, ce qui en limite l'utilisation générale.

La plupart des tests au PdS sont immunochromatographiques et basés sur le flux latéral, et par conséquent, ils offrent des résultats faciles à lire en aussi peu que 30 minutes, sans exiger une longue formation ou de l'équipement spécialisé. Ils sont particulièrement avantageux dans les régions éloignées qui ont un accès limité à des tests en laboratoire centralisés ou une infrastructure locale limitée en matière de laboratoires. Les mêmes lignes directrices décrites plus haut pour les analyses moléculaires au PdS s'appliquent aux analyses sérologiques aux PdS. L'utilisation dans de telles conditions exige une attention et un effort particuliers pour assurer le contrôle et l'assurance de la qualité, comme la participation à une évaluation externe de la qualité, afin de maintenir des tests de haute qualité. De même, il faut prendre des dispositions pour maintenir des données appropriées et des dossiers de qualité pour les résultats des tests aux $\mathrm{PdS}$ avant leur mise en œuvre de façon routinière.

\section{Assurance de la qualité externe}

Tous les laboratoires qui mettent en œuvre des tests pour le SRAS-CoV-2 devraient le faire conformément aux règlements sur les laboratoires médicaux en place dans leur administration. Comme c'est le cas pour d'autres tests cliniques microbiologiques, ils doivent s'inscrire à des programmes d'évaluation de la qualité externes qui peuvent être accédés à l'échelle provinciale, nationale ou internationale. C'est particulièrement important lorsqu'ils assurent le dépistage $d$ 'un agent pathogène émergent comme le SRAS-CoV-2. Le développement et la fourniture de panneaux sérologiques standardisés pour appuyer la mise en œuvre et les tests de compétence seront des éléments clés pour assurer le succès de la mise en œuvre des essais sérologiques dans les laboratoires canadiens.

\section{Détection d'autres virus respiratoires}

L'émergence de la COVID-19 arrive à un moment où beaucoup de régions de l'hémisphère nord traversent leur période de virus respiratoire saisonnière et certaines données laissent entendre la possibilité de co-infection; cependant, les répercussions cliniques de la co-infection sur les résultats pour les patients ne sont pas claires. On s'attend qu'avec la circulation généralisée du virus, les capacités diagnostiques des laboratoires peuvent être dépassées et qu'il soit nécessaire de suspendre certains des services, ou d'avoir recours aux plans d'urgence, ce qui rendrait irréaliste de s'attendre à des tests routiniers généralisés pour les autres virus. Cependant, la détection de la grippe, particulièrement chez les patients devant être hospitalisés ou ceux qui ont des maladies concomitantes entraînant un risque de complications pour eux, devrait continuer d'aider à guider la prise en charge des patients à l'aide d'agents antigrippaux.

\section{Considérations en matière de biosécurité}

Le SRAS-CoV-2 est un agent pathogène du groupe de risque (GR) 3. La propagation ou la culture du virus est restreinte aux laboratoires qui ont des installations de NC3 sous le régime fédéral. Le SRAS-CoV-2 est transmis par la propagation de gouttelettes respiratoires et, à ce titre, les prélèvements respiratoires seraient considérés comme des sources possibles du virus. Bien qu'il y ait des données limitées qui laissent entendre que le SRAS-CoV-2 peut être détecté dans le sang et les selles, il n'y a pas de données pour le moment qui laissent supposer que ceux-ci sont une source d'infection. Il est possible de continuer de mener des activités diagnostiques non propagatrices à l'aide de prélèvements qui n'entraînent pas la concentration ou l'extraction de l'agent pathogène, comme l'analyse chimique de routine, I'hématologie ou l'analyse d'urine, pourvu que l'on prenne les précautions standards. Les prélèvements respiratoires des patients soupçonnés d'être atteints de la COVID-19 peuvent être traités en toute sécurité dans des installations de NC2 avec des précautions supplémentaires, notamment : porter un sarrau de laboratoire, des gants et une protection oculaire pour manipuler les prélèvements préliminaires; effectuer la centrifugation des prélèvements préliminaires dans des godets de sécurité scellés, ou dans des rotors, qui sont chargés et déchargés dans une enceinte de sécurité biologique (ESB) de catégorie II ou un autre dispositif de confinement primaire; utiliser une ESB de catégorie II certifiée ou autre dispositif de confinement primaire pour les procédures qui peuvent produire des aérosols infectieux, y compris le pipetage; et porter une protection respiratoire qui offre un niveau de filtration de $95 \%$ 
ou plus (p. ex. N95) lorsque les activités de production d'aérosols ne peuvent pas être confinées dans une ESB ou autre dispositif de confinement primaire.

Il est recommandé que les laboratoires effectuent une évaluation du risque locale sur les activités comportant des prélèvements provenant de patients atteints de la COVID-19 pour déterminer si d'autres précautions s'imposent.

On ne devrait pas effectuer de culture virale sur des prélèvements respiratoires dans un laboratoire NC2 lorsqu'un agent pathogène nouveau ou émergent est soupçonné, car il s'agit d'agents pathogènes du GR3. On peut envisager la culture virale si le prélèvement a fait l'objet d'un test pour ces agents pathogènes et qu'il est négatif par méthode rRT-PCR.

\section{Désinfection}

Selon les données probantes actuelles, les désinfectants chimiques qui sont efficaces contre les virus enveloppés sont convenables pour la décontamination du SRAS-CoV-2, pourvu qu'ils soient utilisés selon les recommandations du fabricant. Il faut accorder une attention particulière au temps de contact approprié (p. ex. 10 minutes), à la dilution (c.-à-d. la concentration de l'ingrédient actif) et la date d'expiration de la préparation de solution active. Ces désinfectants efficaces incluent l'hypochlorite de sodium (eau de Javel), l'éthanol à $70 \%$, le peroxyde d'hydrogène à $5 \%$, les composés d'ammonium quaternaires et les composés phénoliques. II est possible que d'autres agents biocides soient moins efficaces ( $p$. ex. le chlorure de benzalkonium de $0,05 \%$ à $0,2 \%$, le gluconate de chlorhexidine à 0,02 \%).

L'hypochlorite de sodium (eau de Javel) à une concentration de 1000 ppm (0,1\%) est recommandé pour la désinfection générale des surfaces, et à 10000 ppm (1\%) pour la désinfection des déversements de sang.

Consultez l'Avis de biosécurité SRAS-CoV-2 de I'ASPC (7) et les Lignes directrices provisoires de l'OMS sur la biosécurité laboratoire liées au nouveau coronavirus (2019-nCoV) (anglais seulement) (4) pour en savoir plus.

\section{Déclaration des auteurs}

Le groupe de travail sur les infections virales respiratoires du Réseau des laboratoires de santé publique du Canada (RLSPC) se consacre à fournir un leadership et des directives sur des sujets qui se rapportent aux agents pathogènes viraux respiratoires, y compris l'intervention en laboratoire aux nouveaux virus respiratoires. Le groupe de travail est formé de responsables des laboratoires de santé publique de partout au Canada.

\section{Conflits d'intérêts}

Aucun.

\section{Remerciements}

Le groupe de travail sur les infections virales respiratoires tient à remercier les membres du secrétariat du Réseau des laboratoires de santé publique du Canada (RLSPC), S. Radons Arneson et $D$. Marcino, pour la coordination de la synthèse du document. Nous tenons aussi à remercier le Conseil des directeurs de laboratoires du RLSPC pour leur révision du document.

\section{Références}

1. Word Health Organization. Coronavirus disease 2019 (COVID-19) Situation Report 65 WHO; 2020. www. magnetmail.net/actions/email_web_version.cfm?ep=SUWFh CwRmEENOSZGC2yUcSbCyNDIzfxRuESmZzmC1hV6D6gsrp mRNviR2xWgwjflHNfEpisYU8RnPs2o4MIn4jwLPeBFAtFidmB q8s2Vxpyc3k6QYzIPbcsRDZOr_NsG

2. Agence de la santé publique du Canada. Maladie à coronavirus (COVID-19): Pour les professionnels de la santé. Ottawa (ON) : ASPC; modifié le 24 avril 2020. https://www.canada.ca/en/public-health/ services/diseases/2019-novel-coronavirus-infection/ health-professionals.html

3. Lin M, Beliavsky A, Katz K, Powis JE, Ng W, Williams V, Science M, Groves H, Muller MP, Vaisman

A, Hota S, Johnstone J, Leis JA. What can early Canadian experience screening for COVID-19 teach us about how to prepare for a pandemic. CMAJ. 2020 Mar 6. pii: cmaj.200305. [Epub ahead of print]. DOI

4. World Health Organization. Laboratory biosafety guidance related to the novel coronavirus (2019-nCoV). Interim guidance. WHO; 12 February, 2020. https://www.who. int/docs/default-source/coronaviruse/laboratory-biosafet y-novel-coronavirus-version-1-1.pdf?sfvrsn=912a9847_2

5. Zou L, Ruan F, Huang $M$, Liang $L$, Huang $H$, Hong $Z$, Yu J, Kang M, Song Y, Xia J, Guo Q, Song T, He J, Yen HL, Peiris M, Wu J. SARS-CoV-2 Viral Load in Upper Respiratory Specimens of Infected Patients. N Engl J Med 2020 Mar;382(12):1177-9. [Epub ahead of print]. DOI PubMed

6. U.S. Food and Drug Administration. FAQs on Diagnostic Testing for SARS-CoV-2. https://www.fda.gov/ medical-devices/emergency-situations-medical-devices/ faqs-diagnostic-testing-sars-cov-2

7. Agence de la santé publique du Canada. SARS-CoV-2 (Severe acute respiratory syndrome coronavirus 2 pour Coronavirus du syndrome respiratoire aigu sévère 2). Ottawa (ON) : ASPC; le 29 février 2020. https://www. canada.ca/fr/sante-publique/services/biosecurite-biosuretelaboratoire/directives-avis-avis-speciaux-matiere-biosecurite/ nouveau-coronavirus-27-janvier.html 